\title{
PERCEPTUAL-MOTOR CONTROL STRATEGIES FOR LEFT-TURN EXECUTION
}

\author{
Rayka Mohebbi, Rob Gray \\ Department of Applied Psychology \\ Arizona State University \\ Mesa, Arizona, USA \\ E-mail: rayka@cox.net
}

\begin{abstract}
Summary: The purpose of the present study was to investigate the perceptualmotor strategies used by drivers during left-turn execution as a means of identifying potential causes of such accidents and to inform the design of left-turn warnings. Twenty-one participants executed a series of left turns in a driving simulator. The initial distance(D) and time to intersection(TTI) of an oncoming vehicle were varied orthogonally to present drivers with a range of different safety margins. The initial distance values ranged from 50 to $150 \mathrm{~m}$, and the TTI values ranged between 4 to $12 \mathrm{sec}$. Accidents occurred on roughly $3 \%$ of the trials, with large individual differences in performance. The most common conditions for accidents to occur involved a large value of $\mathrm{D}(>=100)$ and a medium-short value of $\operatorname{TTI}(<=6)$. The timing of left-turn initiation was significantly affected by both the TTI and the distance of the oncoming vehicle. On average, left turns were initiated at a shorter TTI when the distance was large. We conclude that since there were several instances in which drivers used the unsafe strategy of basing their decision to turn on the distance of the oncoming vehicle, a left-turn warning that is based on temporal information alone (e.g., Nowakowski, 2006) will likely be unreliable, because the decision to turn is not solely based on temporal information.
\end{abstract}

\section{INTRODUCTION}

Some of the most dangerous driving accidents are those involving turning vehicles, especially vehicles making left turns. This is mainly due to the fact that during left-turn executions, the relative impact forces are high, and therefore the propensity for injury and damage is great (Caird \& Hancock, 2002). Of the total of 9.7 million single and two-vehicle crashes in the USA during the year 2004, approximately 11 percent $(1,026,000)$ involved a left-turning vehicle. In fact, leftturn accidents resulted in 2,984 fatalities, which compromise 6.0 percent of all U.S. fatalities for that year. Left-turn crashes also accounted for 12.7 percent of all injuries $(350,000)$ and 9.8 percent of all property-damage-only accidents $(673,000)$ for 2004 (NHTSA, 2006).

Turns across the paths of oncoming vehicles compose the overwhelming percentage of left-turn accidents (Staplin and Lyles, 1991). Of the estimated 42.7 percent of intersection-related crashes in the U.S., about 27.5 percent of the intersection-crossing-path crashes fall into the category known as Left Turn Across Path with Opposite Direction Traffic, also known as LTAP-OD. (Najm, Smith, and Smith, 2001). In this crash type, a subject vehicle (SV) approaches a green light and intends to turn left. Due to the lack of a protected left turn arrow, the SV driver must 
decide when it is safe to turn. Often this involves a decision as to whether or not there is a sufficient gap to complete the left turn in front of the oncoming vehicle (Nowakowski, 2006).

The purpose of the present study was to investigate the perceptual-motor strategies used by drivers during LTAP-OD left-turn execution as a means of identifying potential causes of such accidents and to inform the design of left-turn warnings. Although previous research on left-turn execution has identified several factors that influence the decision to turn, including oncoming vehicle type (e.g., car, truck, or motorcycle), temporal gap and approach speed (reviewed in Caird \& Hancock, 2002), the exact strategy drivers are using for left-turn execution is not clear from these studies.

\section{METHODS}

\section{Participants}

Twenty-one drivers between the ages of 19 and 41 participanted in the study. All participants were licensed drivers with 2-20 years of driving experience and were compensated for their participation.

\section{Apparatus}

We used a fixed-base driving simulator composed of two main components: (i) a steering wheel mounted on a table top and pedals (Wingman Formula Force GP, Logitech ${ }^{\mathrm{TM}}$ ) and (ii) a 70-deg horizontal X 52-deg vertical display of a simulated driving scene. The visual scene was rendered and updated by DriveSafety ${ }^{\mathrm{TM}}$ driving simulator software running on two PC's (Dell Optiplex GX270). It was projected onto a wall $2.4 \mathrm{~m}$ in front of the participant using a LCD projector (Hitachi CPX1200SER) and was updated at a rate of $60 \mathrm{~Hz}$ in correspondence with the movement of the simulated car. The simulated driving scene is shown in Figure 1.

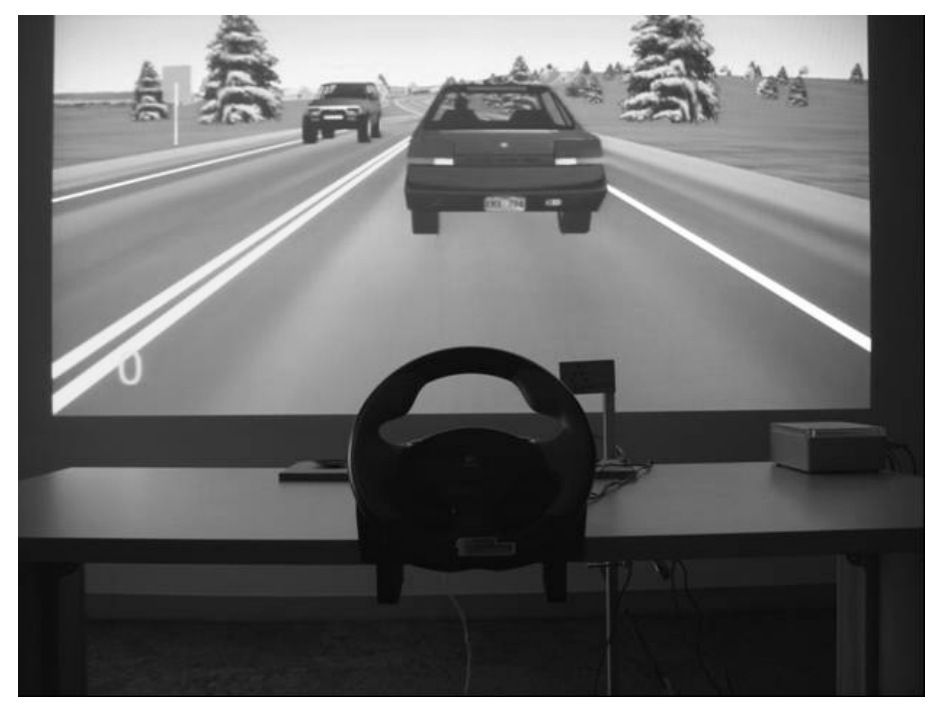

Figure 1. View of the Driving Scene 


\section{Procedure}

Each participant drove through a minimum of 2 practice driving sessions to become comfortable with maintaining a speed of approximately $55 \mathrm{MPH}$, and also to become familiar with the driving course. Once the participant reached a minimum level of mastery in handling the driving simulator, they drove through 5 driving scenarios. The scenarios consisted of a series of signalled intersections, each with an oncoming vehicle. The initial distance (D) and time to intersection (TTI) of this vehicle were varied orthogonally to present drivers with a range of different safety margins. There were 25 different left-turn conditions, representing all possible combinations of 5 values of D $(50,75,100,125$ and $150 \mathrm{~m})$ and 5 values of TTI $(4,6,8,10$, and $12 \mathrm{sec})$ presented in random order. These combinations resulted in oncoming car closing speeds ranging between 4-38 $\mathrm{m} / \mathrm{s}$ (10-83 $\mathrm{mph})$. Participants were instructed to turn in front of the oncoming vehicle if they judged that it was safe to do so, or otherwise to wait to turn until after oncoming car had gone through the interesection. Unlike many of the previous studies, we did not require that the participant come to a stop before completing the turn. The order of the driving scenarios executed by the participant was counterbalanced to prevent learning/practice or order effects interfering with the actual perceptual effects.

\section{Data Analysis}

Our main dependent variable of interest was the TTI difference (TTI-diff) at which the driver iniated the left turn. The TTI-diff was defined as Participant TTI - Oncoming Vehicle TTI. Positive values of this variable indicate that the driver intiated the turn after the oncoming vehicle passed through the intersection, while negative values indicate the turn was made in front of the oncoming vehicle. Mean TTI-diff values were analyzed using a $5 \times 5$ repeated measures ANOVA with TTI and Distance as factors. We also calculated the $\%$ of turns initiated in front of the oncoming vehicle for each of the 25 conditions. Huynh-Feldt corrections for sphericity were used.

\section{RESULTS}

There were a total of 525 left-turn opportunities, of which 14 resulted in accidents $(3 \%)$. The most common (93\%) condition for accidents to occur involved a large( $>=100)$ oncoming vehicle initial distance (D) and a medium-short $(4,6,8)$ value of oncoming vehicle Time to Intersection (TTI) . There was only one case of an accident involving a shorter $\mathrm{D}(75)$ and a medium TTI(8).

Figure 2 shows the Mean TTI-diff at initiation of left turn as a function of the oncoming vehicle TTI for different Initial Distances(D). It should be noted that for Mean TTI-diff values in the range +1 to -1 , the particpant's vehicle is coming very close to the oncoming vehicle during leftturn execution. As expected, there was a significant main effect of TTI with left turns made in front of the oncoming vehicle (negative values of TTI-diff) for long TTIs and made behind the oncoming vehiclefor short TTIs, $F(3,68)=232.195 \mathrm{p}<.0001$. There was also a significant main effect of oncoming vehicle initial distance (D) as on average, left turns were initiated at a shorter TTI when the distance was large, $F(4,80)=6.42 \mathrm{p}<.0001$. As can also be seen in Figure 2, there was a significant TTI $x$ Distance interaction $F(8,162)=2.598 p<.05$, due to the fact that Distance had a larger effect on the timing of turn initiation for short values of TTI than it did for longer 
TTI values. Post-hoc tests revealed that the Mean TTI-diff in the $50 \mathrm{~m}$ condition was significantly higher than in the $150 \mathrm{~m}$ condition for TTI values of $4 \mathrm{t}(20)=6.66 \mathrm{p}=0.000002,6 \mathrm{t}(20)=3.90$ $\mathrm{p}=0.0009,8 \mathrm{t}(20)=3.06 \mathrm{p}=006$ and $10 \mathrm{t}(20)=2.37 \mathrm{p}=0.03 \mathrm{sec}$, but it was not significantly different for TTI value $12 \mathrm{t}(20)=-0.62 \mathrm{p}=0.54 \mathrm{sec}$.

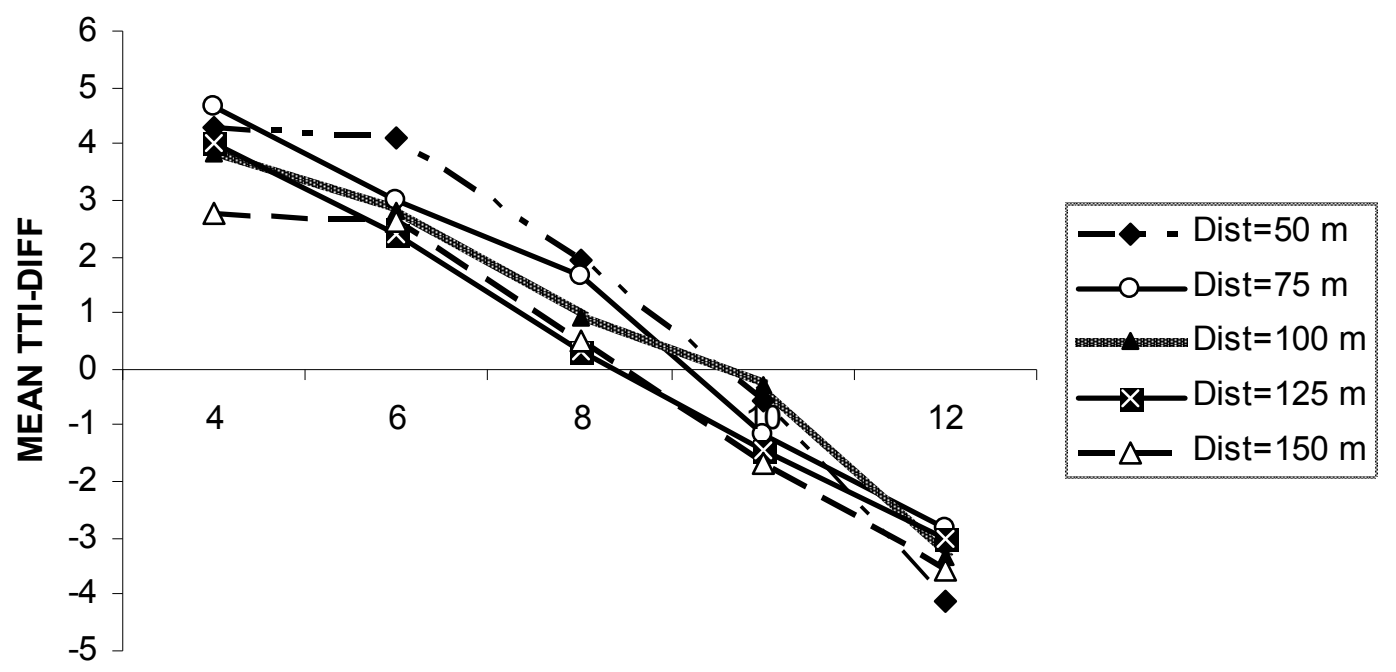

TTI (sec)

Figure 2. Mean TTI-Diff at left-turn initiation

Figure 3 shows the \% of left turns initiated in front of the oncoming vehicle as a function of initial distance (D) of the oncoming vehicle for each value of TTI. This graph shows that the \% left turns made in front of the oncoming vehicle is consistantly larger for higher values of TTI. It also indicates the percentage of left turns go up as D increases within the same TTI value, except for TTI $=4$. There were not any left turns made in front of the oncoming vehicle when TTI $=4$.

The interesting fact highlighted by Figure 3 is the interaction that exists between TTI and D as far as \% left turns are concerned. Though \% left turns generally increases for larger D values (except for $\mathrm{TTI}=4$ ), the manner in which this percentage changes is very much dependent on the TTI value. For higher TTI values of 10 and 12, the increase in \% left turns is more gradual and uniform, while for the short-midrange values of 6 and 8, a sharper increase is observed in the percentage of left turns attempted before the oncoming vehicle. 


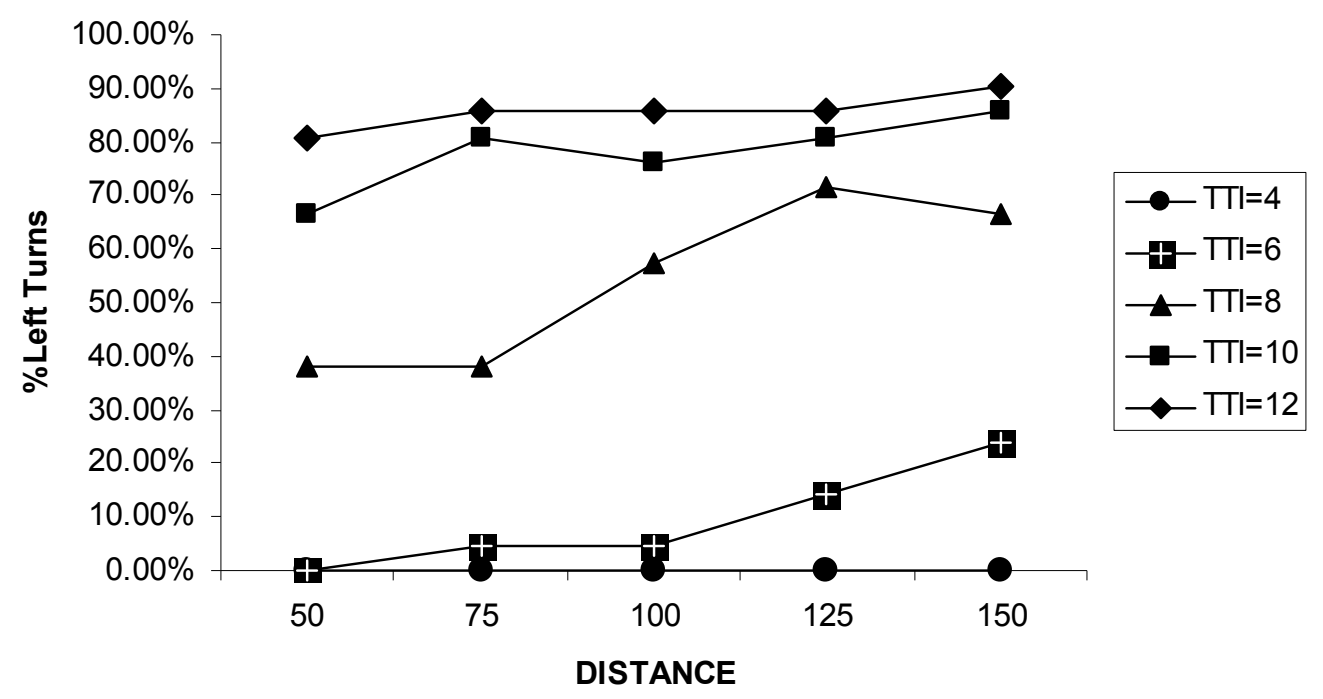

Figure 3. Percentage of left-turns made in front of the oncoming vehicle as a function of Distance (m)

Figure 4 shows the $\%$ of left turns iniated in front of the oncoming vehicle as a function of TTI for different Initial Distance (D) values. This figure re-emphasizes the interesting effect of distance on left-turn execution discussed above. For the low and high values of TTI, where the decision to make the turn in front of the vehicle is presumably easier for the driver (e.g., drivers made zero turns in front of the oncoming vehicle for $\mathrm{TTI}=4$ and $>80 \%$ turns in front for $\mathrm{TTI}=12$ ), there is less effect of distance. However, for mid-range values of TTI, especially for TTI=8, a much more significant effect of distance is observed. For TTI $=8$, the $\%$ left turns is as low as $30 \%$ for small distances and as high as $75 \%$ for larger distances.

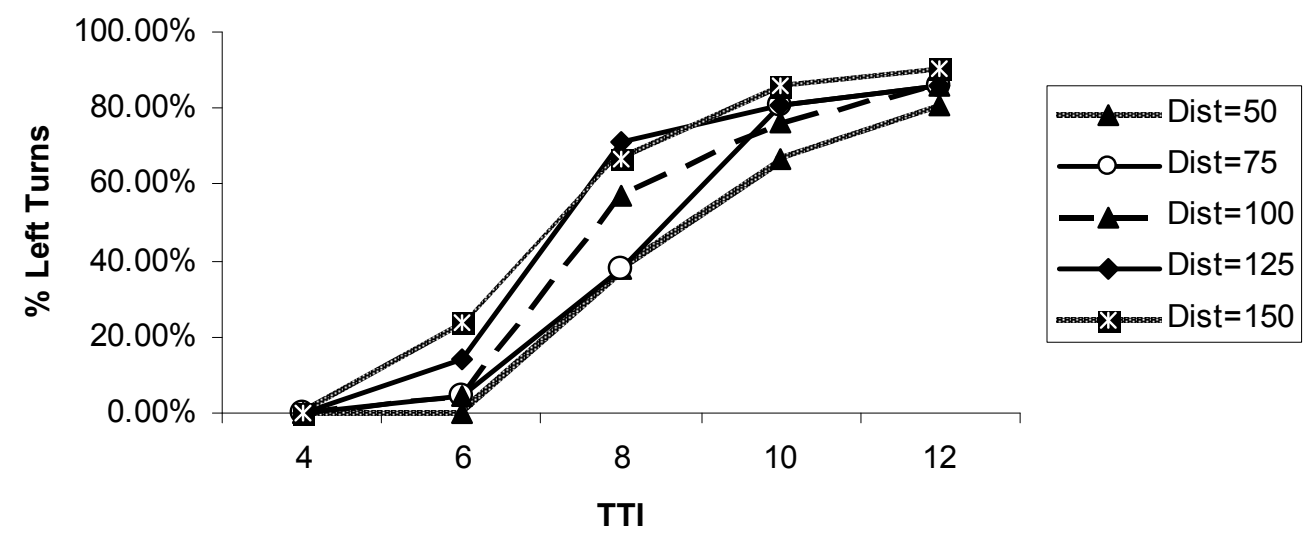

Figure 4. Percentage of left-turns made in front of the oncoming vehicle as a function of TTI (sec) 


\section{CONCLUSIONS}

What perceptual-motor control strategies do drivers use when executing a left-turn? As illustrated in Figure 5, the only safe and robust strategy for performing this maneuver is a temporal-based one (Gray 2005): the driver should only iniate the turn when the TTI of the oncoming car is greater than the time required to complete the left turn (TRT). Basing the decision to turn on either the distance or speed of the oncoming vehicle alone could lead to many unsafe turns (e.g., when the distance is large but the speed is fast).

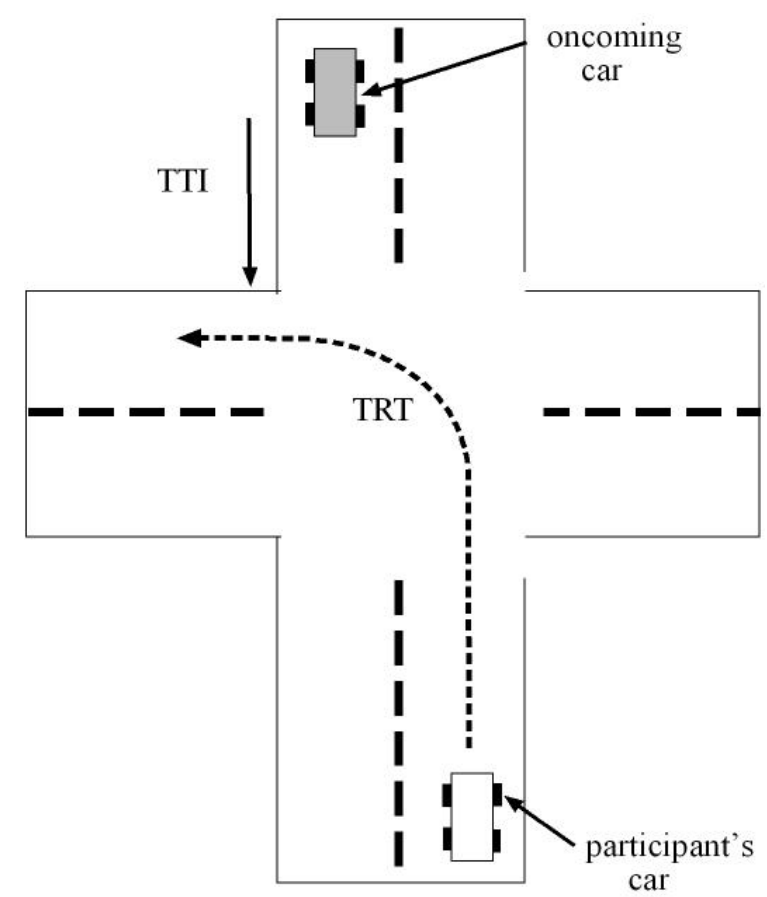

Figure 5. Temporal control strategy for left-turn execution

In the present study, we show that drivers do not use this optimal temporal-based strategy but instead base their decision both on the TTI of the oncoming vehicle and its distance. This was particularly the case for mid-range values of TTI $(6-8 \mathrm{sec})$, where the decision about whether it is safe to iniate a left turn is more difficult for the driver. In these situations drivers appear to use the unsafe strategy of initiating a left turn when the oncoming vehicle's distance is greater than some critical value, irregardless of its TTI. Indeed, the combination of a mid-range TTI and a large distance was the most commom condition for producing an accident in our study. A similar effect of distance has been reported for decisions about overtaking and passing a lead vehicle (Gray \& Regan, 2005).

The effect of oncoming vehicle distance on left-turn execution could provide an explanation for some previous findings in this area. As first reported by Hancock, Caird, Shekhar and Vercruyssen (1991), drivers execute more turns in front of small approaching vechiles such as motorcycles than they do in front of large vehicles such as delivery trucks, even when the approach speed is identical. The previous explanation for this effect was that drivers feel less threatened by small vehicles and are more apt to turn in front of them. We would argue that this could also be explained by the effect of distance on the decision to execute a left turn. Because a 
motorcycle subtends a smaller visual angle, drivers may erroneously perceive that it is a car a large distance away and feel that is safe to turn because the distance is large (see Stewart, Cudworth \& Lishman (1993) for a similar proposal regarding participant accidents).

The use of this distance strategy for left-turn execution is a dangerous problem that needs to be addressed both with driver training and/or driver assistance systems. Driving simulations and/or filmed driving scenarios could be used during driver training to make drivers aware of dangerous left-turn situations in which their perception and decision making may not be reliable (i.e., midrange TTI combined with large oncoming vehicle distance). It would also be beneficial to develop left-turn warnings (e.g., Nowakowski, 2006) that could warn drivers when they initiate a turn in one of these dangerous situations.

\section{REFERENCES}

Caird, J.K., \& Hancock, P.A. (2002). Left-turn and gap acceptance crashes. In: R.E. Dewar and P. Olson (Eds.). Human factors in traffic safety. Tucson, AZ: Lawyers \& Judges Publishing, 613-652.

Gray, R. (2005). The use and misuse of visual information for "go/no-go" decisions in driving. Hennessy, D. \& Wiesenthal, D. (Eds.).Contemporary Issues in Traffic Research and Road User Safety. New York: Nova Science Publishers, 125-133.

Gray R., \& Regan, D. (2005). Perceptual processes used by drivers during overtaking in a driving simulator. Human Factors, 47, 394-417.

Hancock, P. A., Caird, J. K., Shekhar, S., \& Vercruyssen, M. (1991). Factors influencing driver's left turn decisions. Proceedings of the Human Factors Society 35 Annual Meeting, Santa Monica, CA. Human Factors Society, 1139-1143.

National Highway Traffic Safety Administration. (2006). Traffic Safety Facts Annual Report 2004. Washington, DC: US Department of Transportation.

Najm, W., Smith, J., and Smith, D. (2001). Analysis of Crossing Path Crashes (DOT HS 809 423). Cambridge, MA: Volpe National Transportation Systems Center.

Nowakowski, C. (2006). A preliminary evaluation of a driver-infrastructure interface for left-turn warning. Proceedings of the Human Factors And Ergonomics Society $50^{\text {th }}$ Annual Meeting, 2453-2457.

Stewart, D., Cudworth, C.J., and Lishman, J.R. (1993). Misperception of Time-to-Collision by Drivers in Pedestrian Accidents. Perception, 22, 1227-1244.

Taplin, L., and Lyles, R.W. (1991). Age differences in motion perception and specific traffic maneuver problems. Transportation Research Record, 1325, 23-33. 